\title{
MUJER Y MATRIMONIO. EL INTERÉS DE LA INQUISICIÓN POR LA PERSECUCIÓN DE LA BIGAMIA ${ }^{1}$
}

\author{
Henar Pizarro Llorente \\ Universidad Pontificia Comillas - IULCE \\ hpizarro@comillas.edu
}

$\mathrm{L}$

as peculiares características del delito de bigamia han despertado el interés de los juristas, pero, en general, no se ha realizado una lectura social de las circunstancias que rodearon esta transgresión referida a las mujeres procesadas por la Inquisición en los tribunales peninsulares. Se trata de una problemática muy rica en perspectivas de análisis a las que nos proponemos realizar una aproximación.

\section{El Concilio de Trento y el matrimonio}

La institución del matrimonio católico es un sacramento. La definición por parte de la Iglesia del matrimonio como un vínculo indisoluble, asentado sobre el consentimiento mutuo de los cónyuges, se remonta al siglo XII. No obstante, su concreción y articulación fue una tarea que los teólogos y canonistas fueron realizando en los siglos posteriores. Si bien este tema se trató en el Concilio de Florencia de 1439, fue en la tercera etapa del Concilio de Trento (1563-1564), durante la sesión XXIV y recogido en el Canon I, donde se estableció de manera inequívoca el carácter sacramental del matrimonio católico. De esta manera se diferenciaba de las novedades incorporadas a la concepción de dicha institución por parte de los protestantes, sustentadas en la negación de su condición de sacralidad. Del

\footnotetext{
1 Este artículo se ha desarrollado dentro del proyecto «De reinos a naciones. La transformación del sistema cortesano (siglos XVIII-XIX)» (HAR2015-68946-C3-1-P), I+D de Excelencia del Ministerio de Economía y Competividad (MINECO), financiado por la Agencia Estatal de Investigación (AEI) y el Fondo Europeo de Desarrollo Regional (FEDER, UE).
}

Edad de Oro, XXXVIII (2019), pp. 159-172, ISSN: 0212-0429 - ISSNe: 2605-3314

DOI http://doi.org/10.15366/edadoro2019.38.008 
mismo modo, el interés de la Corona por la institución del matrimonio estribaba en que era la base fundamental del orden familiar y estamental, así como garante de las implicaciones sociales y económicas derivadas de la unión, por lo que se realizaron ordenamientos legales para su protección. Por ello, se trató de buscar una definición jurídica común del matrimonio y de realizar un reparto de competencias. Las cuestiones referidas a la validación del matrimonio (promesa, separación y anulación) quedaron reguladas de forma exclusiva por la Iglesia, mientras que los temas relacionados con la propiedad (dotes, contratos matrimoniales, etc.) se situaron bajo la jurisdicción civil. Sin embargo, en aquellos delitos que suponían menoscabo para el matrimonio (concubinatos, matrimonios clandestinos, adulterio, bigamia, etc.), se mantuvo un fuero mixto, es decir, podían intervenir las dos jurisdicciones (Espinar Mesa-Moles 2013: 18-24).

Así pues, la Iglesia reforzó el carácter sacramental del matrimonio en las disposiciones tridentinas, fundamentado en el libre consentimiento de las partes, que se debía de celebrar oficiado por un sacerdote y con testigos. Había de estar precedido de tres amonestaciones públicas, realizadas durante la misa mayor, y solo se podría llevar adelante si no contravenía los impedimentos especificados, como podía ser, entre otros, el grado de parentesco entre los contrayentes. Así mismo, se insistió en la indisolubilidad del vínculo entre los cónyuges, que solo quedaba sin validez tras la muerte de uno de los mismos. Únicamente tras enviudar se podía realizar un segundo matrimonio. Por supuesto, la bigamia se colisionaba con esta disposición. Aun cuando el matrimonio realizado no cumpliese todos los requisitos fijados por el Concilio de Trento, los tribunales eclesiásticos tendieron a ratificar la unión si era público y notorio que la pareja vivía o habían vivido como marido y mujer, no habían sido coaccionados y contaban con edad suficiente, y un sacerdote había sancionado el matrimonio en presencia de testigos. La asamblea conciliar también dispuso que los párrocos habían de llevar un registro de las bodas que celebrasen. Sin embargo, se reiteró la nulidad de los matrimonios clandestinos y la prohibición del concubinato. Igualmente, se admitieron como únicas causas de disolución del matrimonio las establecidas por el pontífice Alejandro III en el siglo XIII, entre las que no se contemplaba el abandono o el adulterio (Espinar Mesa-Moles 2013: 45-47).

El entendimiento del Santo Oficio en el delito de bigamia estuvo directamente relacionado con lo anteriormente expuesto, puesto que la comisión del mismo atentaba contra un sacramento, pero además se podría inducir la conexión con un delito más grave como era el de herejía si se ponía en relación con la interpretación que realizaban los protestantes. Esta cuestión es fundamental, puesto que, con anterioridad a las disposiciones conciliares, se encargaban los tribunales civiles o eclesiásticos bajo su jurisdicción, pero fue tras la celebración del Concilio de Trento cuando se intensificó el control inquisitorial y se adoptaron medidas contundentes contra los infractores (Gacto Fernández 1987: 467-475; Lavenia 2018: 352-354). 
Por otra parte, se contó con otra pieza esencial, puesto que, como hemos señalado, entre las medidas tomadas por el Concilio se encontraba la obligatoriedad de llevar un registro de los matrimonios celebrados en los libros parroquiales. Efectivamente, el aumento de los pleitos por causa matrimonial sufrió un marcado ascenso en los decenios posteriores a la reunión conciliar. La clarificación del modelo matrimonial condicionó que se evidenciasen las irregularidades existentes respecto al mismo. Muchos de estos pleitos obedecían al arraigo popular de prácticas anteriores, como la promesa matrimonial o palabra de matrimonio, si bien estas causas, cuando llegaban al tribunal eclesiástico, se podía comprobar que, frecuentemente, la palabra dada tenía la intención real de estuprar a la mujer, principales víctimas de la ruptura tanto por el engaño como por la soltería. No en pocas ocasiones, hubo mujeres que utilizaron este medio, aludiendo a una promesa que nunca se produjo, para impedir el matrimonio con otra del hombre que ellas habían elegido para sí. No obstante, para el tema que nos ocupa, sin duda tienen más interés los pleitos referidos a las causas de separación y nulidad, puesto que constituían las únicas opciones para poder disolver el vínculo del matrimonio. Se optaba por la nulidad cuando cabía dudar de la forma en que se había contraído el matrimonio o no había sido plenamente realizado, mientras que en la separación la existencia del matrimonio no se cuestionaba. La nulidad conllevaba que, al no existir el matrimonio, las partes quedaban libres de casarse nuevamente, pero, en el caso de la separación, el intento de terminar con el matrimonio estaba imposibilitado por la indisolubilidad del vínculo decretada por el Concilio de Trento, por lo que solo se permitía que afectase a lecho, mesa, habitación y bienes, pero, evidentemente, la pareja estaba imposibilitada para realizar una nueva unión con otras personas. Tras la reglamentación conciliar, resultaba dificultoso obtener la nulidad, por lo que fueron más abundantes los pleitos de separación. Estas separaciones, voluntarias y de mutuo acuerdo, o involuntarias al producirse el abandono, se encuentran en no pocas ocasiones detrás de la comisión del delito de bigamia. Con ánimo de combatir algunos aspectos de esta cuestión, los párrocos quedaron encargados de realizar las amonestaciones públicas previas a la celebración de cualquier matrimonio, así como a poner especial cuidado en casos de extranjeros o de personas cuya profesión implicase una alta movilidad (Campo Guinea 1994: 377-386).

A la Inquisición no le interesaba demasiado el comportamiento sexual de los adultos bautizados, puesto que este tema era asunto de confesionario o pertenecía al ámbito pastoral. El Santo Oficio intervenía cuando en las prácticas conscientes realizadas al margen del matrimonio se pudiese detectar una conexión con creencias heréticas. En el caso de la bigamia, el Santo Oficio trataba de detectar la creencia de que el matrimonio se podía realizar de manera simultánea con más de una persona, o de forma sucesiva sin que se hubiese alcanzado el estado de viudez, y que ello no contravenía la ortodoxia católica (Torquemada Sánchez 2011: 109-112). 
Ciertamente, el afán inquisitorial por entender sobre este delito en detrimento de la jurisdicción civil estaba relacionado con esta cuestión específica, puesto que la posibilidad de la comisión por un delito de carácter herético estaba implícita en la naturaleza del mismo, pero no se podía establecer de manera unívoca. Por ello, los inquisidores trataban de conocer la intención real que había motivado en última instancia la consecución de esta transgresión a través de los interrogatorios, que procuraban indagar y profundizar en la intención última que había impulsado el segundo matrimonio. En el caso de las mujeres bígamas, en general no parece que albergasen ideas heterodoxas en torno al matrimonio ni evidenciaron habitualmente errores de fe. Fueron las circunstancias, las motivaciones afectivas, económicas y sociales, las que se encontraron detrás de sus segundas nupcias (Molina 2017: 34-35).

\section{LA CONSIDERACIÓN DE LOS INQUISIDORES}

Cuando se abordan los temas relacionados con la labor procesal del Santo Oficio, se suele tratar el número de víctimas, la tipología de los delitos, los ciclos en la actividad desplegada por los distintos tribunales, etc. No obstante, suelen pasar desapercibidas cuestiones que están implícitas en esta actuación como la ponderación que los propios inquisidores realizaban en la aplicación de las penas prescritas para cada delito. Quizás sea una excepción, pero, en lo que respecta a la bigamia, las mujeres recibieron unas sentencias condenatorias con castigos menos rigurosos que los hombres, que, como es conocido, conformaban el grueso de los procesados en esta categoría. Ciertamente, los inquisidores contaban con un margen de arbitrariedad a la hora de dictar sentencia, que les permitía emplear distintos grados de castigo en función de la condición del reo y de las circunstancias en que se cometió el delito (Gacto Fernández 2012: 148; Collantes de Terán de la Hera 2017: 75-77). Las penas aplicadas fueron siempre severas, en consonancia con la gravedad del delito, conformando una combinación que incluía la abjuración de levi, las sanciones pecuniarias, los azotes, la vergüenza pública y el destierro. Mientras que los hombres eran condenados a la dura pena de galeras, este castigo estaba vetado para las mujeres, que, en muchos casos, cumplieron parte del destierro confinadas en hospitales o refugios (Galende Díaz 1987: 4865). Esta cierta benignidad por parte de los inquisidores estuvo relacionada con la consideración de la mujer respecto al varón como un ser más débil, vulnerable y, por tanto, menos responsable. Por ello, fueron condenadas en un porcentaje menor que los hombres, puesto que se consideraba que su delito había sido inducido o no obedecía plenamente a su intención (Collantes de Terán de la Hera 2017: 55-58). No obstante, esta situación no debe de confundirse con una actuación clemente. Hemos de considerar que la pena de exilio era especialmente dura para las 
mujeres. Separadas del varón que, en general, les procuraba el sustento y del entorno familiar, con el estigma de haber sido procesadas por la Inquisición, se veían abocadas a la marginalidad social y a afrontar serias dificultades para alcanzar la simple subsistencia (Collantes de Terán de la Hera 2018: 4-6).

Los procesos conservados nos presentan a mujeres que son conscientes de la comisión de un delito, pero que, también muchas de ellas, se han visto abocadas a ello por los condicionantes sociales vinculados a su naturaleza de mujer. Podríamos clasificar a las bígamas en dos grupos. En primer lugar, estarían aquellas que el primer marido se ausentó del hogar conyugal y, pasados los años, la esposa no tiene certeza de que esté vivo, pero tampoco de que esté muerto. Sin duda, el viaje a América daba la oportunidad ideal para que los hombres incurriesen en este delito y, en cierta manera, predispusiesen a su esposa al mismo (Torres Aguilar 1997: 117-122). La lejanía favorecía la ruptura con el pasado, con la tierra dejada y del matrimonio contraído en ella. A veces no se trataba de picaresca, pues existe una casuística que refleja la pérdida de comunicación con la esposa, lo que le llevaba a considerarse viudo o que el matrimonio era de alguna forma inválido, y decidir empezar una nueva vida. Esta situación también ocurría con los regresados a la península, quienes no volvían necesariamente a su lugar de origen. Evidentemente, en estos casos, no hay una dimensión herética (Torquemada Sánchez 2011: 110). Quizás resulta distintivo en el caso americano, como se ha puesto de manifiesto en diversos estudios, que el castigo impuesto a las mujeres que cometían bigamia era de mayor contundencia que el aplicado a los varones, quienes habitualmente no sufrían penas infamantes como sucedía en el caso de las bígamas. Además, fueron sometidas a una diversidad de prácticas condicionadas por su procedencia social. Es decir, eran mucho más drásticas si la mujer era pobre, esclava o indígena. Como hemos señalado, esta contundencia no suele ser la tónica general en los tribunales peninsulares, donde hemos encontrado repetida la siguiente fórmula en los procesos consultados: «Hallamos atentos los autos y méritos del dho proceso que si el rigor del derecho huvieramos de seguir la pudieramos condenar en muchas y muy graves penas, mas queriéndolas moderar con equidad y misericordia por algunas causas y justos respetos que a ello nos mueve...».

Más revelador es el ingente número de peticiones que recibieron las Reales Audiencias para que se obligase a volver al marido ausente, tras su marcha a las Indias, para el cumplimiento patrimonial de sus obligaciones familiares o, lo que resulta más llamativo, pedían que les aclarase cuál era su estado civil una vez que sus maridos habían celebrado un segundo matrimonio, del que tenían noticia por conocidos comunes o cuando se producía la denuncia por bigamia (Arauz Mercado 2012: 74-75).

Evidentemente, no era necesario marcharse a América para poner tierra por medio entre los cónyuges. Así, en segunda instancia, estarían los casos de las 
mujeres que deciden abandonar su localidad de residencia, principalmente impelidas por la imposibilidad de proseguir manteniendo la convivencia por motivos diversos (malos tratos, abusos, etc.). Al cambiar su lugar de residencia, aparecían ante su nuevo entorno como solteras o viudas en caso de ir acompañada por sus hijos, fruto de su primer matrimonio. Efectivamente, la comunidad de acogida mostró habitualmente una opinión favorable hacia la procesada por bigamia, abundando los testimonios sobre su honestidad de vida o la calificación de que se trataba de una «buena mujer». No obstante, muchas de ellas, para dejar constancia de que no tenían una concepción herética del matrimonio y evitar la acción inquisitorial, decidieron regresar con el primer marido, lo que para los inquisidores era suficiente y satisfactorio respecto a su entendimiento del delito (Gacto Fernández 1987: 487-488; Crespo Mas 2014: 76-77; Collantes de Terán de la Hera 2018: 6). En algunos casos, también se constata que estas mujeres accedieron a un segundo matrimonio por la presión social, puesto que procedían a las segundas nupcias después de haber estado amancebadas o simplemente por un impulso de supervivencia económica para ella y sus hijos, para abandonar la prostitución, etc. (García Fuentes 1982: 212-216).

\section{Polémica académica \\ Y PROBLEMÁTICA SOCIAL EN EL PERIODO POSTCONCILIAR}

Ciertamente, la bigamia fue un delito cuya comisión fue mayoritariamente masculina. Así se desprende de los datos que arrojan las fuentes utilizadas en diversos estudios de archivos, como el Diocesano de Cuenca o el Archivo Histórico Nacional, entre los siglos XVI y XVIII. Los procesos por bigamia protagonizados por mujeres corresponden a un $17 \%$ del total (Fernández Carrasco 2015: 180-181; Espinar Mesa-Moles 2013: 360). Sin embargo, un simple acercamiento a estas cifras pone de manifiesto que, durante la primera mitad del siglo XVII, el procesamiento de mujeres por bigamia fue anecdótico, puesto que se registra un número muy pequeño de casos en proporción a los decenios precedentes y, sobre todo, en relación a los habidos en el siglo XVIII, lo que ha provocado que la mayoría de los trabajos hayan optado por este marco cronológico dieciochesco, prestando menos atención al periodo anterior. Si bien se puede colegir que la labor pastoral y la aplicación de los decretos tridentinos habían calado en la sociedad y, por ello, el número de infractoras aminoró, lo cierto fue que confluyeron una serie de circunstancias que pudieron influir en dicha relajación procesal.

Por una parte, la bigamia se trataba, como hemos referido, de un delito en el que podían entender distintas jurisdicciones. A comienzos del siglo XVII, el conocimiento de las causas fue motivo de enfrentamiento entre la justicia episcopal y 
el Santo Oficio en mayor medida que con la justicia civil, si bien, como hemos señalado, el entendimiento de la Inquisición se impuso sobre las mismas. Dicha polémica adquirió ciertas dimensiones en el Santo Oficio portugués, puesto que se vio envuelta por el enfrentamiento existente entre el Inquisidor General y el arzobispo de Lisboa. No obstante, en 1612, el cardenal Arrigoni respaldó la actuación inquisitorial recordando a los prelados lusos que el entendimiento de dicho delito correspondía al Santo Oficio en detrimento de la jurisdicción ordinaria (López-Salazar 2010: 161-176).

Por otra parte, esta cuestión venía a coincidir con la agudización de los problemas surgidos respecto a las competencias en el gobierno de la Inquisición española que se iniciaron tras la muerte del inquisidor general Gaspar de Quiroga en 1594. Destacado miembro de la facción «papista», había frenado las pretensiones del Consejo de Inquisición en la obtención de unas facultades de las que carecía en función de las atribuciones papales. La polémica sobre la ampliación de la potestad del Consejo de Inquisición para convertir el gobierno de la Inquisición en un órgano colegiado en detrimento de los poderes privativos del Inquisidor General concedidos por el pontífice se prolongó durante todo el reinado de Felipe III. Paralelamente, se fue produciendo una evolución en los componentes del propio Consejo y de los miembros de los tribunales de distrito en consonancia con la transformación de los grupos de poder en la Corte, dando cabida a aquellos más proclives a seguir los designios papales. Es decir, los miembros del Consejo de Inquisición se fueron alejando del modelo generado por los miembros del «partido castellano» que habían dominado esta institución los últimos decenios del siglo XVI (Martínez Millán 2008: 263-267).

Esta cuestión no resulta baladí si tenemos en cuenta que fue Melchor Cano quien defendió la sacralidad del matrimonio negada por los reformadores en la referida sesión del Concilio de Trento. No obstante, los teólogos y juristas postridentinos hubieron de desarrollar los acuerdos conciliares y dotarlos de mayor definición, asumiendo también la tarea de enlazar con el pensamiento anterior al Concilio. En este sentido, una de las cuestiones que centraron las discusiones postridentinas fue el reconocimiento por parte de la Iglesia de la nulidad de ciertos matrimonios, por ejemplo, de los clandestinos, como había definido el Concilio, o cuando concurriesen determinadas circunstancias, lo que generaba una contradicción con el principio dogmático de la indisolubilidad. Entre los estudiosos que se ocuparon del tema del matrimonio podemos destacar al jesuita cordobés Tomás Sánchez. Teólogo dogmático, moralista y agudo jurista, se ocupó junto al también jesuita Roberto Belarmino y al dominico salmantino Pedro de Ledesma de recoger la herencia tridentina sobre dicha cuestión. Sánchez escribió la obra Sancto Matrimonii Sacramento, estructurada en diez libros y publicada en tres volúmenes entre 1603 y 1605. Mereció el elogio del papa Clemente VIII 
por la claridad y exactitud con las que abordaba las polémicas existentes en torno a este tema y se convirtió en una referencia obligada en los siglos posteriores (Carrodeaguas 2003: 118-123). Para el problema de la bigamia, sin duda, el libro X, que versa sobre el divorcio, tiene especial interés. El propio autor hace uso del vocablo «divorcio», pero entendido como separación, admisible en los casos de comportamiento depravado, adulterio, amencia y sevicia o malos tratos. Ciertamente, el matrimonio era sustentado en su dimensión sacramental y, por ello, indisoluble. Sin embargo, admitían que el matrimonio podía ser estudiado desde la realidad del contrato o desde la perspectiva social como institución (Carrodeaguas 2003: 144-148). Estas cuestiones fueron muy polémicas y desataron encendidos debates. Esencialmente, la cuestión que se tenía que considerar era probar si, para los bautizados, el matrimonio natural mediante contrato externo válido y el sacramento estaban inseparablemente unidos. Melchor Cano se oponía al automatismo de la forma sacramental del contrato del matrimonio. En contra de sus argumentos se manifestaron el dominico Pedro de Ledesma y los jesuitas Belarmino y Suárez (Tejero 1970: 154-158). Tomás Sánchez se mostró conforme con ellos y elaboró una interpretación propia. La fama internacional alcanzada por Sánchez antes de la publicación del tratado era significativa. La controversia sobre este delicado tema le trajo complicaciones a pesar de que la obra había sufrido diversas censuras en el seno de la propia Compañía de Jesús antes de salir de la imprenta. Se retiraron descripciones de prácticas sexuales que, si bien el autor incluyó para orientar a los confesores, se encontraron excesivamente realistas e indecorosas. El propio general Aquaviva, preocupado por la generación de nuevas polémicas dada la situación en la que se encontraba la Orden, le pidió en reiteradas ocasiones que moderase cualquier aspecto u opinión novedosa que pudiese resultar controvertible. Sánchez realizó las pertinentes retractaciones siguiendo las instrucciones recibidas desde Roma (Lozano Navarro 2000: 79-89). Las tesis de Sánchez y del resto de los teólogos señalados fueron discutidas por el agustino Basilio Ponce de León, sobrino del autor de La perfecta casada, cuyo libro sobre esta cuestión veía la luz en Salamanca en 1624. Las principales discrepancias se centraron en la materia y forma del sacramento del matrimonio, la separabilidad del contrato y del sacramento, y la elevación del matrimonio de los infieles a sacramento cuando reciben el bautismo (Carrodeaguas 2003: 180-182; Olivares D'Angelo 2000: 17-89).

Posiblemente, los inquisidores estuvieron al tanto de estas discusiones entre teólogos y moralistas, pero, sin duda, la importancia que se otorgaba a esta cuestión se percibe de manera más clara cuando transciende el ámbito académico para ponerse de relieve como problema social en la obra de Lope de Vega. Su novela La prudente venganza, publicada en los primeros años del reinado de Felipe IV, abordaba una situación muy reconocible por responder a una realidad. 
En la trama, una joven pareja dispuesta a contraer matrimonio se ve separada por unas circunstancias pergeñadas intencionadamente. La marcha del joven Lisardo a América dejaba a Laura en espera de su retorno. Por una serie de engaños, Laura pensó que Lisardo se había casado en México, por lo que ella accedió a unirse en matrimonio con otro hombre. Descubierta la trampa, ambos se unieron a pesar de los recelos de ella, puesto que esta acción se apartaba de su condición de casada virtuosa. A través de estos amores adúlteros, Lope estaba poniendo en consideración la validez de un matrimonio fruto de un enredo premeditado y que conllevaba una convivencia poco «amorosa», según se afirma en el texto, lo que se relacionaba con la ausencia de hijos. El esposo ultrajado planeó el asesinato de su esposa adúltera de la manera que se acostumbraba a proceder para que permaneciese en secreto el verdadero motivo del crimen. Esta solución violenta, que se produjo con frecuencia en estos años de manera especial en Sevilla, reparaba la ofensa de la honra. Precisamente, este fue uno de los asuntos que más preocupó al jesuita Tomás Sánchez, que trató de manera profusa estas casuísticas. También Pedro de Ledesma se ocupó de denunciar el ejercicio de la violencia por parte de algunos padres para forzar un matrimonio no deseado por sus hijos como sucedía en la obra de Lope (Redondo 2003: 733-744; Ruiz Sastre 2016: 373-444).

\section{Las bígamas ante el tribunal de Toledo (1625-1660)}

Los únicos cinco procesos seguidos en el tribunal inquisitorial de Toledo durante la primera mitad del siglo XVII vienen a ejemplarizar lo expuesto hasta aquí. Sin duda, el proceso más llamativo fue el protagonizado por María González, alias Mariana de los Reyes. Natural de Santarem, se casó en dicha localidad con un trabajador del campo con quien tuvo un hijo. Abandonó a ambos para acudir a Madrid con Francisco de San Antonio, berberisco afincado en dicha ciudad. Según declaró la procesada, cuando llegó a Madrid, se hizo pasar por hebrea, pero expresó su deseo de vivir y morir en la fe católica, por lo que fue bautizada por segunda vez en la Capilla Real, puesto que en la documentación constaba que fue bautizada con anterioridad en Valencia de Miño. Tomado el nombre de Mariana de los Reyes, se casó con el dicho Francisco, puesto que, según afirmaba, había recibido noticia de la muerte de su primer marido. Culpaba de todo el enredo a su segundo esposo, a quien atribuyó la autoría de esta trama para obtener alguna ventaja o merced del rey por su «conversión», mientras que ella se presentaba ante los inquisidores como un ser débil e ignorante con la voluntad sometida a las órdenes cursadas por este hombre al que calificaba de gran embustero. Por otra parte, este caso enlazaba con todos los tópicos referidos a la herencia judeoconversa. Tras el 
párrafo referido a la misericordia que el tribunal quería usar con ella, la sentencia pronunciada recogió las penas habituales: portar las insignias de casada dos veces, la abjuración de levi, el destierro por ocho años del distrito inquisitorial y cien azotes (AHN, Inq., 25, exp. 18). También parece que hubo cierto engaño en el proceso a María González de Candame, desarrollado entre 1628 y 1629. Casada en primeras nupcias con Pedro Rodríguez Calo, alabardero de la Real Audiencia de La Coruña, su marido fue conocedor que «la buena pieza», apelativo con el que nombraba a su mujer, se había vuelto a casar en Madrid tras haber abandonado la ciudad gallega hacía unos años, según refirió un testigo, «por que su marido la yba a la mano». Si bien los testimonios representaron que se trataba de una mujer que «algunos desordenes hazía», ella afirmó que se había vuelto a casar al considerarse viuda. No obstante, la documentación conservada se ve interrumpida después de que los inquisidores ordenasen las pesquisas para atestiguar su nuevo matrimonio con el carnicero Alonso de Torres (AHN, Inq., 25, exp. 18).

Casos muy diferentes fueron los dos siguientes, puesto que terminaron con la anulación del segundo matrimonio. En 1610, Alonso González, tendero de la villa de la Huerta, se casó con María Hernández, quien afirmaba ser viuda. Su matrimonio fue declarado nulo y el hecho no tuvo mayores consecuencias porque María Hernández volvió a hacer vida maridable con su primer marido. Los inquisidores del tribunal toledano suspendieron la causa, aunque las pesquisas se reanudaron en 1635 como consecuencia de los sucesivos matrimonios contraídos por ambos con otras personas posteriormente (AHN, Inq., 25, exp. 13). En 1645 se inició el proceso contra María Hernández, ama en el Hospital de los Niños Expósitos de Madrid. Su segundo marido, Pedro Rodríguez, criado del Conde de Lemos, solicitaba la nulidad de su matrimonio con la citada María ante la audiencia arzobispal. Estando ausente en Granada, su esposa había vuelto con su primer marido, Jacome López de la Cruz, soldado regresado tras prestar servicio de armas, que había retornado para recoger a su esposa y viajar a Italia. Si bien los inquisidores ordenaron que se detuviese a la bígama, no hay constancia de su consecución (AHN, Inq., 26, exp. 3).

El último proceso en el arco cronológico definido fue el de Inés Carrero, que se inició en 1660. Tras sufrir prisión en la Cárcel Real en Madrid, pasó a la jurisdicción inquisitorial por haberse casado en primeras nupcias con Pedro del Campo en 1635 , con quien convivió pocos años y de quien tuvo un hijo. Él acudió a su Galicia natal para comprar unas caballerías, pero no volvió. Criada en una casa de posadas, el cambio del negocio le llevó a trabajar de lavandera y, considerándose viuda, volvió a casarse en 1642. Tras más de veinte años, Pedro del Campo retornó a Madrid. Un testigo aludió en su declaración a que ella pidió «le hechase con el Demonio, que para que benia». Cuando los inquisidores dictaron la sentencia, incorporaron la fórmula de benignidad reiterada, y su condena fue similar a 
la referida en el caso de Mariana de los Reyes, aunque con una pena de destierro reducida a cuatro años (AHN, Inq., 23, exp. 16).

Si bien los casos expuestos se ajustan a las prácticas más comunes, la cortedad de su número durante la primera mitad del siglo XviI denota que la labor inquisitorial no fue significativa en la persecución de este delito en lo que respecta a las mujeres. Más interesante nos parece que, en los últimos decenios de la centuria, declinase la abundante literatura moral a la que hemos hecho referencia. A pesar de que perdiese auge en el contexto de cambio de paradigma que tuvo lugar en el citado periodo, no desapareció la producción cultural destinada a favorecer la implantación de un modelo femenino que generase una mujer eminentemente obediente y sumisa al varón. Como fruto de la cultura barroca, estas obras trataban de ratificar los valores básicos del sistema social, donde, como hemos señalado, el matrimonio constituía una pieza esencial. Sin embargo, el número de procesos inquisitoriales contra bígamas sufrió un aumento considerable.

Así pues, podemos concluir que, independientemente del lugar que ocupase la mujer en este triángulo, sea la bígama o una de las dos esposas, casi siempre estamos ante el vértice más débil. Tanto en los procesos reflejados como en la mayoría de los casos estudiados, las bígamas procesadas no son herejes en cuanto que no albergaban creencias erróneas o vinculadas a doctrinas heréticas, ni tampoco tenían un comportamiento transgresor. Sin duda, los inquisidores tuvieron en consideración las circunstancias que rodearon la comisión del delito.

\section{BIBLIOGRAFÍA}

Arauz Mercado, Diana (2012). «La normativa hispano medieval y su aplicación en Indias: condición femenina dentro del marco penal y procesal». Estudios de Historia de España, 14, pp. 69-82.

Campo Guinea, María del Juncal (1994). «Los procesos por causa matrimonial ante el tribunal eclesiástico de Pamplona en los siglos XVI y XVII». Príncipe de Viana, 55, pp. 377-390.

Candau Chacón, María Luisa (2011). «Literatura, género y moral en el barroco hispano: Pedro de Jesús y sus consejos a señoras y demás mujeres». Hispania Sacra, 63, pp. 103-131.

Carrodeaguas, Celestino (2003). La sacralidad del matrimonio. Doctrina de Tomás Sánchez, S. J. Madrid: Universidad Pontificia Comillas.

Collantes de TerÁn de la Hera, María José (2017). «La mujer en el proceso inquisitorial: hechicería, bigamia y solicitación». Anuario de Historia del Derecho Español, 87, pp. 55-87.

Collantes de Terán de la Hera, María José (2018). «El sexo y la Inquisición». ILCEA, 33 $<$ https://journals.openedition.org/ilcea/pdf/4986> [Consulta: 20/02/2019]. 
Crespo Mas, Teodoro (2014). «"Para castigo suyo y exemplo de otros”. El procés de fe per bigàmia contra la benissera Anna Maria Yvars Castelloli (1644)». Aguaits, 34, pp. 69-80.

Espinar Mesa-Moles, María Paz (2013). Jurisdicción penal ordinaria e Inquisición en la Edad Moderna (a propósito del delito de bigamia). Fernando Suárez Bilbao (dir.) [tesis doctoral]. Madrid: Universidad Rey Juan Carlos $<$ https://eciencia.urjc. es/handle/10115/12097> [Consulta: 10/03/2019].

FERNÁNDEZ CARRASCO, Eulogio (2015). «El comisario en las primeras diligencias de investigación en el delito de bigamia. El caso de Manuel Riñón del Mozo en el tribunal de la Inquisición de Cuenca». Revista de Derecho UNED, 16, pp. 173-215.

GaCto Fernández, Enrique (1987). «El delito de bigamia y la Inquisición española». Anuario de Historia del Derecho Español, 57, pp. 465-492.

Gacto Fernández, Enrique (2012). Estudios jurídicos sobre la Inquisición española. Madrid: Dyckinson.

Galende Díaz, Juan Carlos (1987). «La Inquisición borbónica toledana. Su intervención en las causas de bigamia». Cuadernos de Estudios Manchegos, 17, pp. 43-67.

García Fuentes, José María (1982). «Inquisición y Sexualidad en el reino de Granada en el siglo XVI». Chronica Nova, 13, pp. 207-229.

LAVENIA, Vicenzo (2018) «Pietro Belo, fiscale del Sant'Uffizio: sugli inizi del'attività della Congregazione». Tiempos Modernos, 37/2, pp. 341-359.

LóPEZ-SAlazAr, Ana Isabel (2010). Inquisición portuguesa y Monarquía hispánica en tiempos del perdón general de 1605. Lisboa: Colibri.

Lozano Navarro, Julián José (2000). Tomás Sánchez. Granada: Editorial Comares.

Martínez Millán, José (2008). «El sistema polinodial. Consejos y facciones cortesanas». En José Martínez Millán y Maria Antonietta Visceglia (dirs.), La monarquía de Felipe III: La Corte. Madrid: Fundación Mapfre, pp. 261-269.

Molina, Fernanda (2017). «“Casada dos veces”. Mujeres e inquisidores ante el delito de la bigamia femenina en el virreinato del Perú (siglos XVI-XVII)». Memoria Americana. Cuadernos de Etnohistoria, 25, pp. 31-46.

Olivares D’Angelo, Estanislao (2000). «Discrepancias doctrinales entre teólogos postridentinos y Basilio Ponce en su tratado De Matrimonio». Archivo Teológico Granadino, 63, pp. 17-89.

REDONDO, Agustín, (2003). «Teatralidad, trayectoria narrativa y recorrido ideológico en una novela de Lope de Vega, La prudente venganza». Homenaje a Stefano Arata, Criticón, 87-89, pp. 733-744 <http://www.cervantesvirtual.com/nd/ark:/59851/ bmc93168> [Consulta: 01/03/2019].

Ruiz SAStre, Marta (2016). Mujeres y conflictos en los matrimonios de Andalucía occidental: el Arzobispado de Sevilla durante el siglo XVII. María Luisa Candau Chacón (dir.) [tesis doctoral] Huelva: Universidad de Huelva $<$ http://rabida.uhu.es/dspace/ bitstream/handle/10272/12496/Mujeres_y_conflictos_en_los_matrimonios.pdf? sequence $=2>$ [Consulta: 10/02/2019].

TeJERo, Eloy (1970). «Significación sacramental y orden jurídico del matrimonio (ss. XIVXVI)». Ius Canonicus, 10, pp. 137-160. 
Torquemada SÁnchez, María Jesús (2011). «Apuntes sobre Inquisición y feminidad en la cultura hispánica». Foro, Nueva época, 14, pp. 101-118.

Torres Aguilar, Manuel (1997). «Algunos aspectos del delito de bigamia en la Inquisición de Indias». Revista de la Inquisición, 6, pp. 117-138.

Recibido: 07/05/2019

Aceptado: 03/07/2019 
MUJER Y MATRIMONIO.

EL INTERÉS DE LA INQUISICIÓN POR LA PERSECUCIÓN DE LA BIGAMIA

RESUMEN: La Inquisición española puso especial interés en poner bajo su jurisdicción la persecución de la bigamia. Si bien se trató de un delito cometido mayoritariamente por hombres, existió un número de mujeres bígamas que merecen atención. En general, fueron las víctimas de una situación social, económica y/o afectiva. En cierta manera, los inquisidores tuvieron en consideración estas cuestiones cuando dictaron sentencia.

PALABRAS CLAVES: bígama, Inquisición, mujeres, control social.

WOMAN AND MARRIAGE.

THE INTEREST OF THE INQUISITIONFOR the PERSECUTION OF BIGAMY

AвSTRACT: The Spanish Inquisition put special interest in placing under its jurisdiction the persecution of bigamy. Although it was a crime committed mainly by men, there were a number of bigamous women who deserve attention. In general, they were the victims of a social, economic and/or affective situation. In a way, the inquisitors took these issues into consideration when they passed a sentence

KeYwords: Bigama, Inquisition, women, social control. 